Enfermagem Brasil 2016;15(3):146-51

\title{
ARTIGO ORIGINAL Instrumento para avaliação do graduando de enfermagem em prova prática
}

Bruno Vilas Boas Dias, M.Sc. ${ }^{*}$, Eula Carolina M. Lemes ${ }^{\star \star}$, Cícera Mayara B. de Oliveira ${ }^{\star \star *}$, Guilherme Conrado Pierazo***, Gláucia Cristina V. Borges ${ }^{\star \star *}$, Leomar dos Santos ${ }^{\star \star *}$

*Enfermeiro, Mestre em Ciências da Saúde pela Faculdade de Medicina de Jundiaí/SP, Especialista em Cardiologia pela UNIFESP, Docente do curso de graduação em Enfermagem do Centro Universitário Padre Anchieta de Jundiaí/SP e Faculdade de Campo Limpo Paulista/SP, ${ }^{*}$ Enfermeira, Especialista em Docência, Docente do curso de graduação em Enfermagem do Centro Universitário Padre Anchieta de Jundiaí/SP, ${ }^{* * *}$ Acadêmicos do Curso de Enfermagem do Centro Universitário Padre Anchieta de Jundiaí/SP

Recebido em 18 de dezembro de 2014; aceito em 21 de agosto de 2015.

Endereço para correspondência: Bruno Vilas Boas Dias, Rua Bom Jesus de Pirapora, 100/140, Departamento de Enfermagem, 13207-270 Jundiaí SP, E-mail: bruno.dias@anchieta.br

\section{Resumo}

Objetivos: Elaborar um instrumento simplificado para avaliação do aluno de enfermagem na realização de prova prática. Métodos: Relato de experiência sobre a elaboração de um instrumento de avaliação para utilização em provas práticas de semiologia e semiotécnica de enfermagem. Foi testado com mais de 400 acadêmicos de graduação. Resultados: 0 instrumento, composto por seis etapas, aborda a técnica a ser realizada pelo aluno, 0 desempenho na execução e na resposta à eventual pergunta sobre o procedimento e também a nota estabelecida. Diante da vasta aplicação, o instrumento foi implementado. Conclusão: Foi possível elaborar um instrumento básico, porém eficiente, pois o professor deixava o aluno desenvolver a técnica e focava somente nos pontos necessários à melhoria. Isso tornou o processo rápido e completo no retorno ao acadêmico sobre seu desempenho seguidamente à prova. Tudo em apenas uma página, o que proporcionou economia de recursos e tempo, sem prejuízo de sua eficácia.

Palavras-chave: avaliação educacional, aprendizagem, laboratórios, estudantes de enfermagem.

\begin{abstract}
Evaluation instrument of nursing student in practical test

Objectives: To develop a simplified tool to evaluate nursing students during practical test. Methods: Experience report aiming at preparing assessment tool for practical tests of semiology and nursing semiotics. It was tested with more than 400 academic students. Results: The instrument is composed of six steps, which concern technique to be used by the student, performance in the implementation and response to any question about the procedure and also the established grade. In view of the wide application, the instrument was implemented. Conclusion: It was possible to draw up a basic instrument, but effective, because the teacher leaves the student to develop the technique and focuses only on points needed to improve. This made the process faster and complete and the student receive feedback on his performance immediately after the test. All on one page, this provided savings in time and resources, without impairing its effectiveness.
\end{abstract}

Key-words: educational measurement, learning, laboratories, nursing students.

\section{Resumen}

Instrumento de evaluación del estudiante de enfermería en la prueba práctica

Objetivos: Desarrollar herramienta simplificada para evaluar competencias en la prueba práctica de estudiantes de enfermería. Métodos: Relato de experiencia sobre elaboración de test para evaluar pruebas prácticas de semiología y semiótica de enfermería. El instrumento fue probado con más de 400 estudiantes de enfermería. Resultados: El instrumento consta de seis pasos y aborda la técnica que el alumno realizará, el desempeño en la ejecución y en la respuesta a cualquier pregunta sobre el procedimiento y también la nota establecida. Dicho 
instrumento se ha implementado debido a la amplia aplicación. Conclusión: Fue posible la elaboración de un instrumento básico, pero eficaz, porque el profesor deja al estudiante desarrollar la técnica y se centra sólo en los puntos que necesitan mejorar. Esto hizo que el proceso fuera más rápido y completo y el estudiante recibe el retorno sobre su desempeño inmediatamente después de la prueba. Todo en una página, que proporciona economía de tiempo y recursos, sin perder eficacia.

Palabras-clave: evaluación educacional, aprendizaje, laboratorios, estudiantes de enfermería.

Introdução

As Diretrizes Curriculares Nacionais do Curso de Graduação em Enfermagem reforçam que o enfermeiro é o profissional com formação generalista, humanista, crítica e reflexiva, qualificado para o exercício de Enfermagem com base no rigor científico e intelectual e pautado em princípios éticos. Para tal, as diretrizes estabelecem que façam parte dos projetos pedagógicos dos cursos de enfermagem competências relacionadas ao conhecimento, habilidades e atitudes, a fim de promover a resolutividade multidisciplinar para o cuidado e promoção da saúde de todos [1].

Por outro lado, as Diretrizes Curriculares visam também a proporcionar às Instituições de Ensino Superior (IES) um direcionamento para a implantação e a implementação dos projetos político-pedagógicos. Entretanto, não são fórmulas prontas, já que o contexto sóciopolítico-cultural que envolve cada IES fala mais alto e exige inovadoras formas de saber, fazer e ser [2].

Compete, portanto, a cada IES planejar e implementar o ensino e avaliar a aprendizagem dos acadêmicos. Fato é que não necessariamente as mudanças precisam ser ou são vultuosas. Na verdade o que se espera de um curso em andamento são ajustes pontuais, reformulações e adequações mediante cada contexto apresentado. Dessa forma, ao preparar os alunos para atividades práticas de sua carreira, surgiu a necessidade de organizar a avaliação a que são submetidos de modo a ficar mais claro para professor e aluno o que precisa ser melhorado.

Dentre os vários conhecimentos adquiridos, os procedimentos como sondagem vesical e acesso venoso, entre tantos outros, exigem dos alunos, durante a graduação, o conhecimento aliado à habilidade e a atitude para algumas situações que se apresentam. As habilidades são aptidões desenvolvidas e que tornam os profissionais diferenciados em alguns aspectos de seus pares [3].

No contexto das aulas em laboratório de práticas assistenciais, o conhecimento é o produto de experiências, de aprendizagem, de busca de informações e de elaborações mentais aplicados objetiva e eficazmente. Para a realização de qualquer técnica é primordial, além do seu conhecimento, no mínimo, o de sua indicação, materiais necessários, possíveis complicações e cuidados de enfermagem.

A prática, o "fazer repetidamente" faz com que o aluno adquira maior desenvoltura, além de maior facilidade, rapidez e segurança na realização do procedimento ou técnica. Aliada ao conhecimento teórico, por fim, gera atitudes que se referem a comportamentos relacionados com aplicação de princípios e valores, ou seja, ação consciente e livre de danos [3].

Desse modo, a enfermagem necessita, mesmo com tantas barreiras e dificuldades, fundamentar-se em evidências, sempre, incluindo resultados de pesquisas, por meio de métodos que os qualifiquem e o validem. Assim, por experiência do professor responsável pela disciplina, optou-se por elaborar um instrumento que pudesse servir de base para desenvolvimento das avaliações práticas em laboratório, com intuito de deixar claro ao aluno sobre o parecer final do professor de forma qualitativa. Ademais, o retorno ao aluno é imediato, documentado e com custo baixo para a instituição, em relação ao instrumento propriamente dito.

Isso posto, entende-se que o aluno imbuído dessa busca também imerge na relação "administração e cuidado", enfermeiro não cuida sem administrar e não administra sem cuidar. Direta ou indiretamente, o acadêmico precisa entender que a realização do procedimento assertivo no tocante à técnica vai consequentemente proporcionar conforto e promoção da saúde, melhora a recuperação do paciente e para a instituição que representará, haverá melhoria de ordem econômica em vários aspectos. 
Assim, para melhor organização e evidência de cada passo na realização dos procedimentos, teve-se como objetivo elaborar um instrumento simplificado para avaliação do aluno de enfermagem na realização de prova prática.

\section{A prática baseada em evidências: história e definição}

Durante a prática clínica surgem os mais variados problemas que precisam ser resolvidos por meio de decisões baseadas nas melhores evidências que emergem dos resultados de pesquisas e ou consenso de especialistas [4,5].

Foi no Reino Unido com o epistemologista Archibald Cochrane, em 1972, que surgiu a Prática Baseada em Evidências (PBE), mas, somente 20 anos depois, a comunidade científica organizou a Cochrane Collaboration, com informações científicas em todos os campos da saúde [6].

O profissional atuante e imerso em seu contexto e adquirindo sua experiência progressivamente tem impacto profundo no processo de desenvolvimento de uma pesquisa, haja vista que novas tecnologias vêm surgindo, a expectativa de vida está aumentando, o perfil de morbimortalidade está sofrendo alterações e consequentemente, o gasto com a saúde vem crescendo em ritmo acelerado no mundo [7,8]. Assim, para a PBE, a experiência ou expertise prévia contribui de forma a ratificar ou refutar uma nova evidência, antes que ela seja incorporada à prática clínica [6].

\section{Prática baseada em evidências como tecnologia para decisões em saúde}

A busca de evidências pode diminuir a margem de erro da assistência e analisar a questão de custo-efetividade, reduzindo gastos e melhorando a assistência [9]. Desse modo, a PBE busca aprimorar decisões oriundas da racionalidade clínica, que tem por base não toda e qualquer evidência científica, mas, somente, aquela decorrente do modo clínico de se fazer pesquisa [6].

Assim, mesmo que os resultados alcançados em uma pesquisa não sejam os melhores ou os esperados, são relevantes e têm uma repercussão importante e benéfica para a ciência, no processo de construção do conhecimento, ao longo da história [10].

\section{A Enfermagem baseada em evidências: histórico e definição}

Na década de 80, surgiu a Pesquisa Baseada em Evidências (PBE), no Canadá, por meio de um grupo de estudos da Universidade de McMaster, que se preocupava com uma adequada avaliação das publicações científicas. Foram publicados diversos textos nesse sentido e, posteriormente, ficaram conhecidos como Medicina Baseada em Evidências (MBE).

A Enfermagem Baseada em Evidências (EBE) teve origem com a medicina baseada em evidências [11], tendo, por sua vez, a definição de uso consciencioso, explícito e criterioso das informações oriundas de teorias e pesquisas para a tomada de decisão sobre o cuidado prestado a indivíduos ou grupos de pacientes, priorizando as necessidades individuais e preferenciais [12], ou seja, está amparada na utilização de resultados de pesquisas e dados que proporcionem a melhoria da qualidade originários de especialistas reconhecidos e de confirmada experiência que comprovem a prática [13].

Em relação à aplicabilidade do termo "evidências" para a enfermagem, na literatura americana é sinônimo de "baseada em pesquisa", enquanto que, no Canadá e Inglaterra, torna-se amplo e está associado à utilização dos recursos da investigação [13]. Independentemente do local o termo "evidências" está ligado à pesquisa, entretanto, para a EBE, o binômio pesquisa e prática são um todo inseparável, significando que evidências e o uso dos resultados da pesquisa na clínica estão interligados à atividade diária do enfermeiro.

A visibilidade e oportunidade de desenvolvimento de pesquisas em Enfermagem, com o apoio de várias áreas da saúde para disseminação na prática clínica e nas vidas diárias de indivíduos e famílias foi preocupação, desde o início, para a Enfermagem. Para solucionar este gargalo, foi criado, em 1986, o National Institute of Nursing Research (NINR), um órgão vinculado ao Instituto Nacional de Saúde $(\mathrm{NIH})$ dos Estados Unidos [14,15].

A Enfermagem reconhece que a pesquisa é a base para o conhecimento e desenvolvimento da profissão [6]. Para tal, busca de forma mais ampla (em relação à MBE), não somente aspectos baseados em pesquisas, mas afirmação dos cinco passos que devem 
ser seguidos: transformar a dúvida em questão clínica; buscar a melhor evidência para respondê-la; avaliar a validade, impacto e aplicabilidade dessa informação; integrar a evidência com a experiência clínica; e as características do paciente e a autoavaliação de desempenho nos passos posteriores [16].

Entretanto, a EBE ainda não tem um caminho próprio, seguindo a MBE, que inicia pela formulação de uma dúvida, que se denomina questão clínica, derivada da prática clínica. Por sua vez, a questão clínica deve conter quatro componentes triviais recomendados pela MBE [17]: a situação clínica; a intervenção; o grupo-controle, que é padrão de comparação; e o desfecho clínico, que é o resultado esperado para a intervenção. Essas estratégias são conhecidas pela sigla PICO [18,19]:

P: definir a população, situação clínica ou problema;

I: definir intervenção;

C: adicionar uma comparação;

O: descrever um desfecho clínico;

A EBE possui pesquisas, em grande número, com características qualitativas, o que, para a PBE, é considerada uma evidência menor. Todavia, a ausência de alta qualidade de evidências não impossibilita a tomada de decisões baseadas nelas. Dessa forma, entende-se que o necessário é a melhor evidência disponível e não a melhor evidência possível [20,21].

Contudo, foram identificadas barreiras para a aplicabilidade da EBE pelos enfermeiros. Um levantamento realizado na Austrália suscitou fatores como: acessibilidade de resultados de pesquisa; resultados antecipados de usar pesquisa; apoio organizacional para usar pesquisa; e apoio de outros para usar pesquisa [22]. Também se ressalta que, a rigor, as evidências levantadas nem sempre são incorporadas à prática clínica, devido a variáveis como o local de trabalho, recursos humanos e financeiros para a implementação das mudanças necessárias e, principalmente, se estão em acordo com o paciente e família [23].

\section{Métodologia}

Relato de experiência de um professor do curso de graduação em enfermagem de um centro universitário do interior do estado de São Paulo. A indagação nasceu de estudos e percepções desse professor, em relação à necessidade de maior clareza e consolidação dos pontos que cada aluno desenvolve na realização de diferentes técnicas, durante as provas práticas.

A instituição onde foi desenvolvido o instrumento conta, atualmente, com três disciplinas relacionadas à técnica (Propedêutica e Semiotécnica I, Propedêutica e Semiotécnica II e Laboratório de Práticas Assistenciais), cujas práticas são realizadas, essencialmente em ambiente de laboratório. Nesse local, são realizados procedimentos práticos relacionados ao exame físico e aos sinais vitais, punção venosa e arterial; sondagens vesical, gástrica, enteral e retal; banho e higienização; curativos; cuidados com drenos, entre outros.

Quando da ocorrência de uma avaliação, o professor solicita ao técnico do laboratório que disponha na bancada do setor, todos os materiais necessários e em número suficiente para a realização de todas as técnicas, conforme previsão. O material não fica agrupado por técnica para não influenciar o aluno, exigindo a evocação de conhecimentos necessários para a concretização da prática em avaliação.

Outro preparo é em relação à avaliação. O professor prepara as técnicas a serem realizadas e praticadas, anteriormente, em forma de pergunta ou caso clínico resumido, em um impresso, as recorta e prepara para sorteio. O aluno entra no laboratório, mediante ordem de lista de presença, e ele próprio sorteia sua técnica. A seguir, seleciona e prepara seu material na bancada (nesse momento, já é informado que está sendo avaliado) e vai até o boneco para realização do procedimento.

Todo esse processo vinha sendo realizado em papel de avaliação tipo folha de pauta ou sulfite. Entretanto, entendendo a necessidade de melhoria nesse registro, o professor dessas disciplinas propôs elaborar um instrumento que fosse prático, simples, organizado e ao mesmo tempo eficaz, ou seja, que tivesse os itens necessários para anotação de toda avaliação sem tomar muito tempo, pois a prova prática, por ser individual, despende tempo mais elevado, quando comparado a outro método avaliativo. 
Desse modo, foi elaborado um instrumento simplificado para avaliação do aluno na realização de procedimentos técnicos de enfermagem (Apêndice 1) por esse professor e aplicado com o auxílio de outra professora, em provas, e também pelos monitores, em simulados de avaliação.

Resultados e discussão seguem:

O instrumento de avaliação elaborado é composto basicamente de seis etapas que

1. Identificação do aluno: Nome, número de registro na instituição e data.

2. Técnica sorteada pelo aluno: Em que o aluno descreve exatamente a técnica que sorteou para fazer.

3. Desempenho na realização da técnica: Campo exclusivo do professor que deve de maneira sucinta elencar os pontos assertivos e falhos do aluno, durante a técnica, e observações pertinentes.

4. Desempenho nas respostas às perguntas teóricas referentes à técnica: Caso o professor opte por fazer perguntas relacionadas à técnica sorteada e executada, este campo possibilita a descrição desempenho do desempenho do aluno na resposta.

5. Nota: O professor informa a nota ao aluno imediatamente, no fim da avaliação. Por meio das anotações, nos campos três e quatro, pode evidenciar ao discente o motivo da nota atribuída, mediante seu critério e desempenho apresentado, inclusive, do ponto de vista de competências, habilidades e atitudes no decorrer da técnica apresentada.

6. Assinatura do aluno: Optou-se por deixar esse tópico para que se tivesse a ciência do aluno, em relação ao seu desempenho e nota já apresentados.

Anualmente, passam pelo laboratório de práticas assistenciais, cerca de 150 alunos do curso de graduação em enfermagem, tendo sido o instrumento elaborado utilizado em avaliações de mais de 400 alunos. Portanto foi testado e aprovado na instituição, após ter sido implementado, em cerca de quatro centenas de avaliações.

Foi possível elaborar um instrumento básico, porém eficiente, pois o avaliador deixa o aluno desenvolver a técnica e foca somente nos pontos necessários à melhoria. Isso tornou 0 processo avaliativo rápido e completo no retorno ao acadêmico sobre seu desempenho seguidamente ao término da prova ou simulação. Tudo em, apenas, uma página, o que proporcionou economia de recursos e tempo, sem prejuízo de sua eficácia.

1. Brasil. Ministério da Educação. Secretaria de Educação Superior. Diretrizes Curriculares para os Cursos de Graduação. Resolução CNE/ CES n ㄱ, de 7 de novembro de 2001. Brasília: Ministério da Educação; 2001.

2. Santana FR, Nakatani AYK, Silva e Souza AC, Casagrande LDR, Esperidião E. Diretrizes curriculares nacionais do curso de graduação em enfermagem: uma visão dialética. Revista Eletrônica de Enfermagem 2005;7(3):295-302.

3. Resende EJ. A força e o poder das competências: conecta e integra: competências essenciais, competências das pessoas, competências de gestão, competências organizacionais. Rio de Janeiro: Qualitymark; 2004

4. Rosenberg W, Donald A. Evidence based medicine: an approach to clinical problemsolving. Br Med J 1995;29;310(6978):1122-6.

5. Madigan EA. Evidence-based practice in home healthcare: a springboard for discussion. Home Healthcare Nurse 1998;16(6):411-5. 
6. Lima DVM, Lacerda RA. Repercussões oxi-hemodinamicas do banho no paciente em estado crítico adulto hospitalizado: revisão sistemática. Acta Paul Enferm 2010;23(2):278-80.

7. Silvia LK. Avaliação tecnológica e análise custo-efetividade em saúde: a incorporação de tecnologias e a produção de diretrizes clínicas para o SUS. Cienc Saude Coletiva 2003;8(2):501-20.

8. French P. The development of evidence-based nursing. J Adv Nursing 1999;29(1):72-8.

9. Drummond JP, Silva E, Coutinho M. Medicina baseada em evidências: novo paradigma assistencial e pedagógico. 2a ed. São Paulo: Atheneu; 2002.

10. Cortella MS. A escola e o conhecimento: fundamentos epistemológicos e políticos. $8 a$ ed. São Paulo: Cortez; 2004.

11. Closs SJ, Cheater FM. Evidence for Nursing practice: a classification of the issues. $J$ Adv Nursing 1999;30(1):10-7.

12. Ingersoll GL. Evidence-based nursing. Nursing Outlook 2000;48(4):151-2.

13. Stetler CB, Brunell M, Guiliano KK, Morsi D, Prince L, Newell-Stockes V. Evidencebased practice and the role of nursing leadership. J Nurs Adm 1998; 28(7-8):45-53.

14. Stevens KR, Cassidy V. Evidence based teaching: current research in nursing education. Sudbury: Jones \& Bartlett; 1999.

15. National Institute of Nursing Research (NINR). [online]. January 2007. [citado 2007 Out 23]. Disponível em URL: http://www.ninr.nih.gov/aboutNINR/NINRDirectorsPage/.

16. Sackett DL, Straus SE, Richardson WS, Roseberg W, Haynes RB. Evidence-based medicine: how to practice and teach. 2 ed. Churchill Livingstone; 2000.

17. Bork AMT. Enfermagem Baseada em Evidências. Rio de Janeiro: Guanabara Koogan; 2005.

18. Richardson WS. Ask, and ye shall retrieve. Evid Based Med 1998;3:100-1.

19. Nobre MR, Bernardo VM, Jatene FB. Evidence based clinical practice. Part 1: wellstructured clinical questions. Rev Assoc Med Bras 2003;49(4):445-9.

20. Muir-Gray JA. Evidence-based healthcare. 2 ed. Edinburg: Churchill; 2004.

21. Galvão MC, Sawada NO, Rossi LA. A prática baseada em evidências: considerações teóricas para sua implementação na enfermagem perioperatória. Rev Latinoam Enferm 2002;10(5):690-5.

22. Retsas A. Barriers to using research evidence in nursing practice. J Adv Nurs 2000;31(3):599-606.

23. Ciliska D, Cullum N, Marks S. Evaluation of systematic reviews of treatment or prevention interventions. Evidence-Based Nurs 2001;4(4):100-4. 